\title{
BMJ Open Assessing the diagnostic accuracy of PCR-based detection of Streptococcus pneumoniae from nasopharyngeal swabs collected for viral studies in Canadian adults hospitalised with community- acquired pneumonia: a Serious Outcomes Surveillance (SOS) Network of the Canadian Immunization Research (CIRN) study
}

\author{
Hayley D Gillis, ${ }^{1}$ Amanda L S Lang, ${ }^{1}$ May EISherif, ${ }^{1}$ Irene Martin, ${ }^{2}$ \\ Todd F Hatchette, ${ }^{1}$ Shelly A McNeil, ${ }^{1}$ Jason J LeBlanc, ${ }^{1}$ on behalf of the Serious \\ Outcomes Surveillance (SOS) Network of the Canadian Immunization Research \\ $(\mathrm{CIRN})$
}

To cite: Gillis HD, Lang ALS, ElSherif M, et al. Assessing the diagnostic accuracy of PCR-based detection of Streptococcus pneumoniae from nasopharyngeal swabs collected for viral studies in Canadian adults hospitalised with community-acquired pneumonia: a Serious Outcomes Surveillance (SOS) Network of the Canadian Immunization Research (CIRN) study. BMJ Open 2017;7:e015008. doi:10.1136/ bmjopen-2016-015008

- Prepublication history and additional material are available. To view these files please visit the journal online (http://dx.doi org/10.1136/bmjopen-2016015008).

Received 3 November 2016 Revised 28 March 2017 Accepted 12 April 2017 CrossMark

For numbered affiliations see end of article.

Correspondence to Dr Jason J LeBlanc; jason. leblanc@nshealth.ca

\section{ABSTRACT}

Study design Detection and serotyping of Streptococcus pneumoniae are important to assess the impact of pneumococcal vaccines. This study describes the diagnostic accuracy of PCR-based detection of $S$. pneumoniae directly from nasopharyngeal (NP) swabs collected for respiratory virus studies.

Methods Active surveillance for community-acquired pneumonia (CAP) in hospitalised adults was performed from December 2010 to 2013. Detection of pneumococcal CAP $\left(\mathrm{CAP}_{\text {Spn }}\right)$ was performed by urine antigen detection (UAD), identification of $S$. pneumoniae in sputum or blood cultures. S. pneumoniae was detected in NP swabs using lytA and $C D S A$ real-time PCR, and serotyping was performed using conventional and real-time multiplex PCRs. For serotyping, the Quellung reaction, PCR-based serotyping or a serotype-specific UAD was used. Results NP swab results were compared against CAP cases where all pneumococcal tests were performed $(n=434)$, or where at least one test was performed ( $\mathrm{n}=1616)$. $\mathrm{CAP}_{\mathrm{Spn}}$ was identified in $22.1 \%(96 / 434)$ and $14.9 \%$ (240/1616), respectively. The sensitivity of NP swab PCR for the detection of $S$. pneumoniae was poor for $\mathrm{CAP}_{\text {Spn }}(35.4 \%(34 / 96)$ and $34.17 \%(82 / 240))$, but high specificity was observed $(99.4 \%(336 / 338)$ and $97.89 \%$ (1347/1376)). Of the positive NP swabs, a serotype could be deduced by PCR in $88.2 \%$ (30/34) and 93.9\% (77/82), respectively.

Conclusions While further optimisation may be needed to increase the sensitivity of PCR-based detection, its high specificity suggests there is a value for pneumococcal surveillance. With many laboratories archiving specimens

\section{Strengths and limitations of this study}

- This study provided the first validation of PCRbased detection and serotyping of Streptococcus pneumoniae directly from nasopharyngeal (NP) swabs collected for viral studies in clinical cases of community-acquired pneumonia (CAP).

- PCR-based detection and serotyping of $S$. pneumoniae from NP swabs were compared with cases of CAP defined by chest radiograph confirmation, compatible symptoms and one or several culture-based, antigen-based, and molecular-based laboratory tests.

- To further characterise the value of this method for $S$. pneumoniae colonisation studies, a direct comparison between culture-based and PCR-based detection of $S$. pneumoniae from the same and other anatomical sites should be explored.

for influenza virus surveillance, this specimen type could provide a non-culture-based method for pneumococcal surveillance.

\section{INTRODUCTION}

Streptococcus pneumoniae is a bacterium that colonises the human oropharynx and nasopharynx and can cause life-threatening infections like community-acquired pneumonia (CAP). ${ }^{1-3}$ Pneumococcal 
polysaccharide and conjugate vaccines have reduced colonisation and the burden of disease caused by some $S$. pneumoniae serotypes, and ongoing surveillance including serotyping of $S$. pneumoniae is important to monitor the impact of pneumococcal vaccines and disease epidemiology. ${ }^{4-8}$

While traditional methods of serotyping (Quellung reaction) require live organism, PCR-based detection and serotyping of $S$. pneumoniae can be performed on a variety of clinical specimens without culture. ${ }^{9-15}$ Our laboratory previously demonstrated the feasibility of these PCR methods on nasopharyngeal (NP) swabs routinely collected for respiratory virus studies. ${ }^{9}$ The serotype distribution mirrored trends obtained with traditional Quellung serotyping, but the PCR methods were not thoroughly validated with specimens collected from patients with pneumococcal disease. ${ }^{9}$

We hypothesise that NP colonisation with $S$. pneumoniae should be detected by PCR in cases of pneumococcal CAP $\left(\mathrm{CAP}_{\mathrm{Spn}}\right)$, and the serotype distribution should reflect those of cultured S. pneumoniae isolates from these $\mathrm{CAP}_{\mathrm{Spn}}$ cases. The objective of this study was to assess the diagnostic accuracy of PCR-based detection and serotyping of $S$. pneumoniae from NP swabs collected for viral studies in clinical CAP cases with laboratory confirmation of $\mathrm{CAP}_{\mathrm{Spn}}$.

\section{MATERIALS AND METHODS \\ Study eligibility}

This study was performed prospectively as part of the Serious Outcomes Surveillance (SOS) Network of the Canadian Immunization Research Network (CIRN) surveillance for pneumococcal disease. ${ }^{16}$ The CIRN SOS Network has been conducting active surveillance for CAP and invasive pneumococcal disease (IPD) in hospitalised adults since December 2010 at nine acute care hospitals spanning five Canadian provinces (British Columbia, Ontario, Québec, New Brunswick and Nova Scotia). ${ }^{16}$ Dedicated surveillance monitors reviewed daily adult admissions (aged $\geq 16$ years) to medical wards or intensive care units (ICUs) to identify patients with an acute respiratory illness, which were admitted from the community or another acute care hospital with influenza virus infection, CAP, asthma or acute exacerbation of chronic obstructive pulmonary disease, or any other respiratory tract infection or symptoms. Patients were eligible for enrolment if they met the study case definition for CAP. Cases were enrolled consecutively from 1 December 2010 to 31 December 2013 if they met the case definitions and consent was obtained.

\section{CASE DEFINITIONS}

A case was considered CAP if a hospitalised patient presented within 72 hours of admission with a new or evolving pulmonary infiltrate on chest radiograph suggesting pneumonia (as interpreted by the treating physician or radiologist), and if there were two or more of the following signs or symptoms of pneumonia: temperature $>38^{\circ} \mathrm{C}$, cough, sputum production, shortness of breath, pleuritic chest pain, crackles or consolidation on chest examination. Admissions from long-term care facilities were excluded. Pneumococcal CAP $\left(\mathrm{CAP}_{\mathrm{Spn}}\right)$ was defined as a CAP case with laboratory confirmation of $S$. pneumoniae using urine antigen detection (UAD) or positive culture from respiratory secretions (sputum or bronchial alveolar lavage). Bacteremic $\mathrm{CAP}_{\mathrm{Spn}}$ was defined as an isolation of $S$. pneumoniae from blood culture in a confirmed CAP case.

\section{ETHICS}

This study was approved by the Research Ethics Boards (REB) at each participating hospital. Eligible patients or their legally authorised representative signed written informed consent for participation in the study.

\section{LABORATORY TESTING}

All specimens and S. pneumoniae isolates recovered from culture at each CIRN SOS hospital were stored at $-80^{\circ} \mathrm{C}$ and shipped in batches on dry ice to the SOS Network central laboratory in Halifax, Nova Scotia. All data were blinded to the laboratory personnel and investigators prior to data analyses, through generation of non-nominal identification codes for each patient and laboratory samples, and importation of data into the Dacima laboratory information system. Sputum and blood specimens were cultured according to routine practices in each CIRN SOS site hospital. Urine specimens were stabilised by adding $25 \mathrm{mM}$ piperazine-N, $\mathrm{N}^{\prime}$-bis (ethanesulfonic acid) (PIPES) buffer, pH 6.8 (Boston BioProducts, Ashland, Massachusetts, USA) prior to freezing. NP swabs were collected in $3 \mathrm{~mL}$ universal transport media (UTM) (Copan Diagnostics) for viral studies and were also subjected to nucleic acid extraction and PCR for $S$. pneumoniae detection and serotyping.

\section{S pneumoniae culture from sputum and blood}

Briefly, S. pneumoniae isolates recovered from blood, or as predominant organisms in sputum, were confirmed by optochin disc susceptibility (Oxoid, Basingstoke, Hampshire, UK) and tube bile solubility analyses using standard laboratory methods. ${ }^{1718}$ All streptococci were cultured at $35^{\circ} \mathrm{C}$ in $5 \% \mathrm{CO}_{2}$ on trypticase soy agar with $5 \%$ sheep blood (Becton Dickinson, Mississauga, Ontario, Canada). Bacterial growth was harvested from overnight cultures and suspended in PCR-grade water to a McFarland value of approximately 1.0 prior to nucleic acid extraction.

\section{Traditional serotyping of $S$. pneumoniae isolates}

Quellung serotyping on S. pneumoniae isolates is the gold standard for serotyping, and was performed at the National Microbiology Laboratory (NML) (Winnipeg, Manitoba, Canada) using commercial pool, group, type and factor antisera, as recommended by the manufacturer (SSI Diagnostica, Statens Serum Institut, Copenhagen, Denmark). ${ }^{19}$ 


\section{Urine antigen detection}

Two UAD assays were used in this study. First, urine was tested using the commercial pan-pneumococcal UAD $\left(\mathrm{UAD}_{\mathrm{Spn}}\right)$ BinaxNOW S. pneumoniae urinary antigen test (Alere Scarborough, Scarborough, Maine, USA) according to manufacturer recommendations. A PCV13-specific assay UAD $\left(\mathrm{UAD}_{\mathrm{PCV13}}\right)$ was performed on a Luminex 2.0 instrument as previously described. ${ }^{5}$ The $\mathrm{UAD}_{\mathrm{Spn}}$ was only able to identify $S$. pneumoniae capsular polysaccharides in urine, whereas the $\mathrm{UAD}_{\mathrm{PCV} 13}$ was able to identify and provide a serotype..$^{5}$

\section{Detection of $S$. pneumoniae from NP swabs}

Nucleic acids were purified from NP swabs (in UTM) using a MagNA Pure Total Nucleic Acid Isolation kit (Roche, Laval, Québec, Canada) on a MagNA Pure LC instrument, as recommended by the manufacturer. Elution volume was set at $100 \mu \mathrm{L}$, and $5 \mu \mathrm{L}$ served as template for all PCR reactions: lyt $A$ and $c p s A$ real-time PCR for the detection of S. pneumoniae, and lytA and cpsA positive NP swabs were subjected to conventional multiplex PCR (cmPCR) or real-time multiplex PCR (rmPCR) for serotyping as previously described. ${ }^{910}$

Real-time PCR for the lytA or cpsA was carried out as previously described..$^{9}$ Briefly, $25 \mu \mathrm{L}$ reactions consisting of: 1× Taqman Universal PCR Master Mix (Life Technologies), $200 \mathrm{nM}$ of primers (LytA-F and LytA-R or CpsA-F and CpsA-R) and $200 \mathrm{nM}$ of probe (LytA-pb or CpsA-pb) (see onlinesupplementary table S1). Amplifications were performed on an Applied Biosystems 7500 Fast instrument (Life Technologies) as follows: $95^{\circ} \mathrm{C}$ for $10 \mathrm{~min}$, 45 cycles of $95^{\circ} \mathrm{C}$ for $15 \mathrm{~s}$ and $60^{\circ} \mathrm{C}$ for 60s. Threshold cycle values were determined using software provided by the manufacturer. Primers and probes were obtained from Integrated DNA Technologies. Since other viridans group streptococci or other organism could potentially generate false positives (FPs) with lytA detection alone, an NP swab was only considered positive for $S$. pneumoniae if both lyt $A$ and $c p s A$ target were amplified. ${ }^{9}$ NP swabs positive only by lytA PCR were considered indeterminate and interpreted as negative for $S$. pneumoniae during method performance evaluation compared with $\mathrm{CAP}_{\mathrm{Spn}}$ cases. ${ }^{9}$

\section{PCR-based serotype deduction}

For rmPCR, amplification conditions were identical to the lytA and cpsA real-time PCR. ${ }^{9}$ Each rmPCR reaction was performed in $25 \mu \mathrm{L}$ reactions consisting of: $1 \times$ Platinum Quantitative PCR SuperMix-UDG (Life Technologies), $50 \mathrm{nM}$ of $\mathrm{MgCl}_{2}$ and primer and probes concentrations and combinations listed in (see online supplementary tables S1 and S2, respectively.

For cmPCR reactions, $25 \mu \mathrm{L}$ reaction volumes consisted of $1 \times$ enzyme mix from the Multiplex PCR kit (Qiagen, Toronto, Ontario, Canada) with primer combinations and concentrations listed in online supplementary tables S1 and S3, respectively. All cmPCR reactions contained primers cmCpsA-F and cmCpsA-R which target the capsule biosynthesis gene A ( $p p s A)$ that is used as an internal control. Amplification was performed in 96-well plates using a C1000 thermocycler (Biorad Laboratories, Mississauga, Ontario, Canada) as follows: $95^{\circ} \mathrm{C}$ for $90 \mathrm{~s}$, 35 cycles of $95^{\circ} \mathrm{C}$ for $30 \mathrm{~s}, 54^{\circ} \mathrm{C}$ for $90 \mathrm{~s}$ and $72^{\circ} \mathrm{C}$ for $60 \mathrm{~s}$, followed by $72^{\circ} \mathrm{C}$ for $10 \mathrm{~min}$. Amplicons were resolved on a $1.2 \%$ agarose gel electrophoresis with $10 \mu \mathrm{g} / \mathrm{mL}$ ethidium bromide staining and visualised using a GelDoc XR+ with ImageLab software V.5.1 (Biorad Laboratories). Expected amplicon sizes in base pairs are denoted in online supplementary table S1. Oligonucleotides were synthesised by Integrated DNA Technologies (Coralville, Iowa, USA).

\section{Modified gold standard}

Since there is no gold standard for the identification of $S$. pneumoniae, results for NP swabs detection of $S$. pneumoniae by PCR was compared with two different modified gold standards: (1) CAP cases with NP swabs were obtained and all four pneumococcal tests were performed (sputum and blood culture, and the two UAD methods) (figure 1A) and (2) CAP cases with NP swabs were obtained and any of the pneumococcal tests were performed (figure 1B). A positive result from any UAD or an isolate of S. pneumoniae recovered blood culture was considered a $\mathrm{CAP}_{\mathrm{Spn}}$ case. For sputum, S. pneumoniae was considered a $\mathrm{CAP}_{\mathrm{Spn}}$ case if the culture was predominant over normal flora. NP swabs PCR for detection waswere classified as true positives (TP), true negative (TN), FP or false negative (FN) based on these comparators. Serotyping in S. pneumoniae-positive NP swabs (by lyt $A / c p s A$ real-time PCR) was compared against cultured $S$. pneumoniae isolates characterised by Quellung serotyping or positive results from $\mathrm{UAD}_{\mathrm{PCV} 13}$ in culture-negative $\mathrm{CAP}_{\mathrm{Spn}}$ cases detected solely by antigen testing (figure 2).

\section{STATISTICAL ANALYSIS}

NP swab PCR was compared against the two modified gold standards to assess performance characteristics (table 1). $\chi^{2}$ with Fisher's exact test was used to assess statistical significance of the method comparison and 95\% CIs were included for each of the reported parameters: sensitivity $(\mathrm{Sn}=\mathrm{TP} / \mathrm{TP}+\mathrm{FN})$, specificity $(\mathrm{Sp}=\mathrm{TN} / \mathrm{TN}+\mathrm{FP})$, positive likelihood ratio ( $\mathrm{LR}+=\mathrm{Sn} / 100-\mathrm{Sp})$; negative likelihood ratio $(\mathrm{LR}-=100 \mathrm{Sn} / \mathrm{Sp})$; pretest probability $(\mathrm{TP}+\mathrm{FN} /$ $\mathrm{TP}+\mathrm{TN}+\mathrm{FP}+\mathrm{FN})$; pretest odds=pretest probability $/(1-$ pretest probability); post-test odds=pretest odds $\times$ LR and the post-test probability $=$ pretest odds $\times$ LR. All analyses were performed using SAS V. 9.4.

\section{RESULTS}

Both CAP and $\mathrm{CAP}_{\text {Spn }}$ were in a patient population of hospitalised adults, most over the age of 65 years with comorbidities and with significant mortality and morbidity (in terms of length of hospital stay, requirement for ICU admission and mechanical ventilation). ${ }^{16}$ Of the 4769 cases of all-cause CAP enrolled from 1 December 2010 to 31 December 2013, 434 had NP swabs for viral 

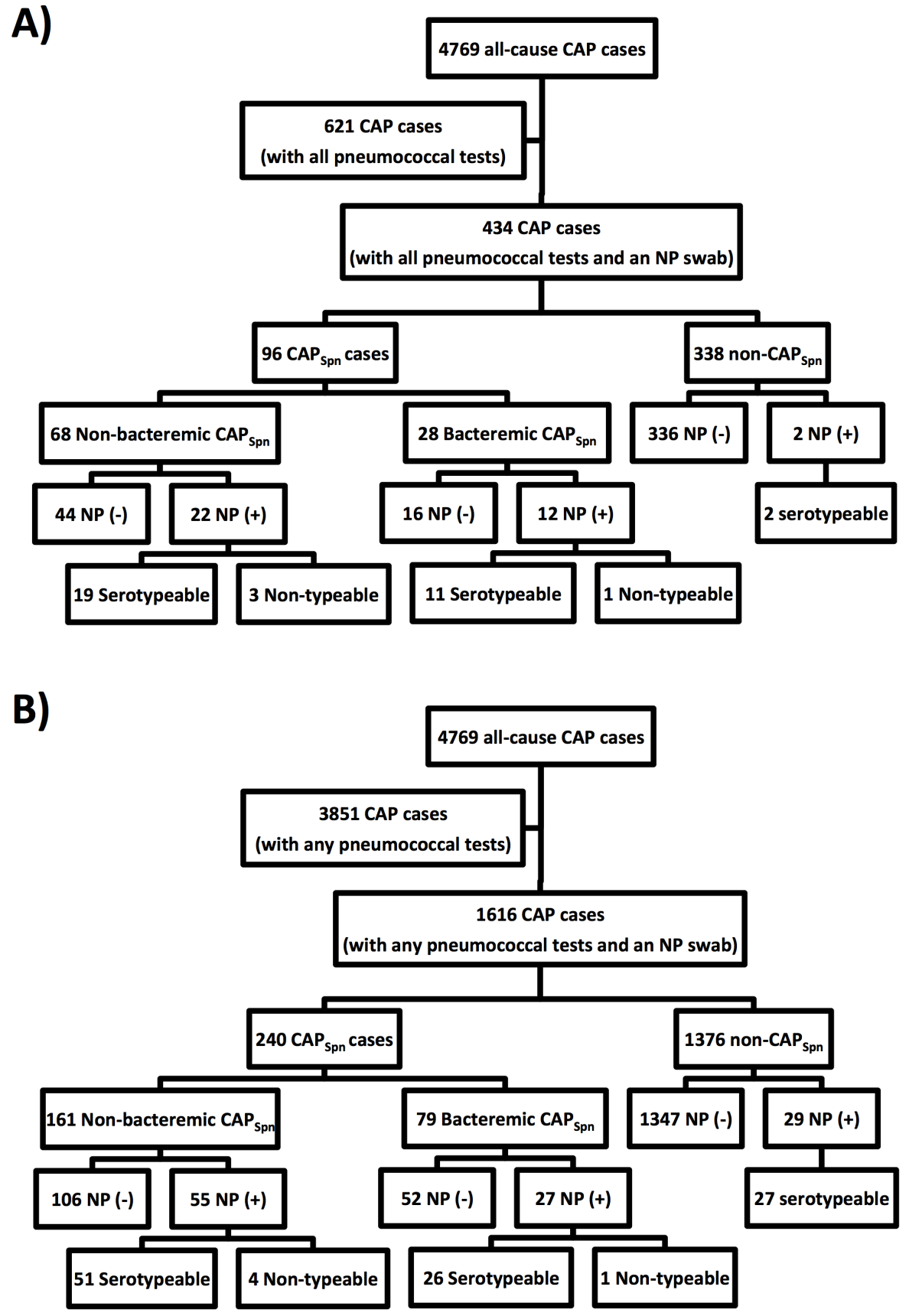

Figure 1 Diagram illustrating the flow of participants and a summary of laboratory results based on disease categories. Community-acquired pneumonia (CAP) cases were considered if a nasopharyngeal (NP) swab was available and all (A) or any (B) of the following tests were performed: sputum culture (SC), blood culture (BC), a pan-pneumococcal urine antigen detection $\left(\mathrm{UAD}_{\mathrm{Spn}}\right)$ and/or a 13-valent pneumococcal conjugate vaccine serotype-specific UAD (UAD $\left.\mathrm{PCV}_{13}\right)$. Pneumococcal CAP $\left(\mathrm{CAP}_{\mathrm{Spn}}\right)$ cases were defined based on any test positive for SC, BC, UAD $D_{\text {spn }}$ or $U_{A D_{P C V 13}}$. Bacteremic CAP $P_{\text {Spn }}$ represents $C A P_{\text {Spn }}$ cases with Streptococcus pneumoniae isolated from blood. NP swab positive (+) or negative (-) results represent the combined results of IytA and cpsA real-time PCR for the detection of $S$. pneumoniae. Serotypeable NP results were evaluated using conventional and real-time PCR-based serotyping. ${ }^{9}{ }^{10}$

studies collected concomitantly with sputum, blood and urine (figure 1A). Of the 434 CAP cases, 22.1\% (96/434) were identified as $\mathrm{CAP}_{\text {spn }}$ (figure 1). Of the $96 \mathrm{CAP}_{\text {Spn }}$ cases, $68(70.8 \%)$ were non-bacteremic $\mathrm{CAP}_{\mathrm{Spn}}$ and 28 $(29.2 \%)$ were bacteremic $\mathrm{CAP}_{\mathrm{Spn}}$ (figure $\left.1 \mathrm{~A}\right)$. The sensitivity for $S$. pneumoniae detection of $\mathrm{CAP}_{\mathrm{Spn}}$ using lytA and cpsA real-time PCR on NP swabs was significantly $(\mathrm{p}<0.0001)$ less than the modified gold standard at $35.4 \%$ (34/96) (table 1). Only two NP swabs were positive in CAP cases not identified as pneumococcal disease (figure 1B), resulting in high specificity and good post-test probability (table 1). 


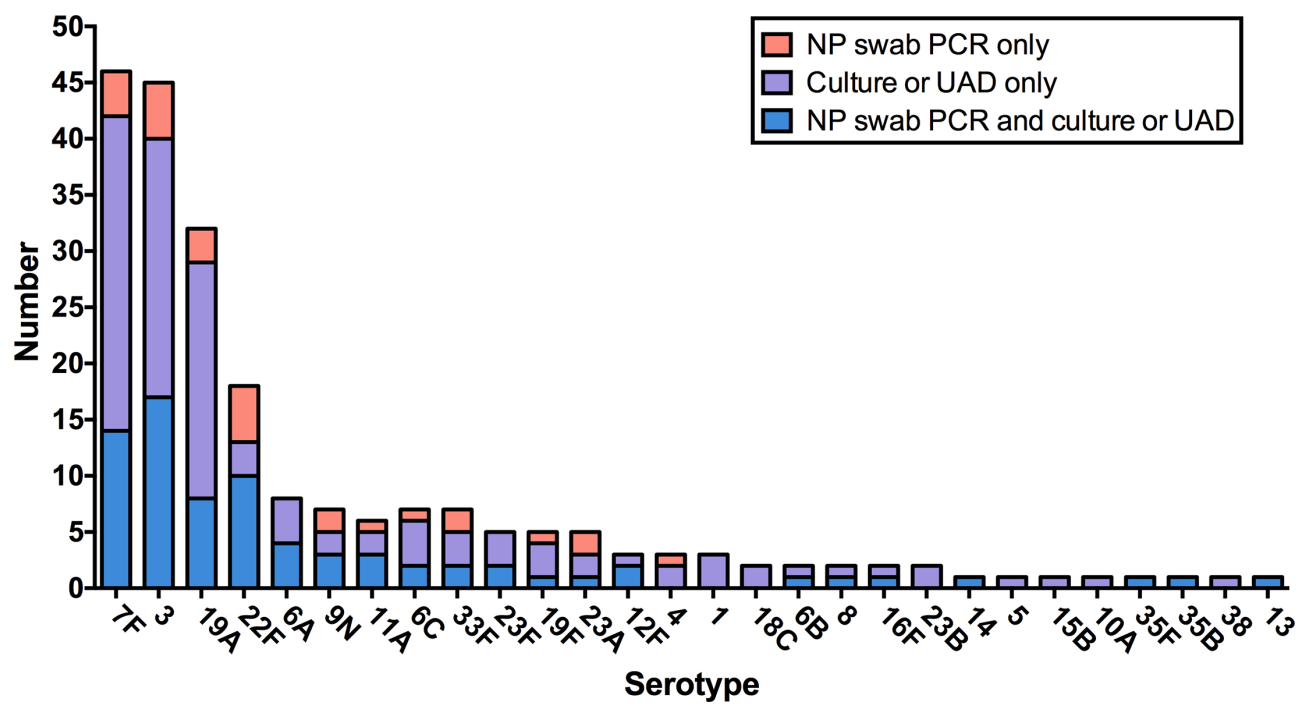

Figure 2 Streptococcus pneumoniae serotype distribution obtained using NP PCR compared with traditional methods. Serotype results were obtained from CAP cases having received at least one pneumococcal tests. NP swab PCR results positive for lytA and $\operatorname{cPs} A$ were subjected to $\mathrm{cmPCR}$ and $\mathrm{rmPCR}$ and compared with S. pneumoniae isolates obtained from culture (sputum or blood) and Quellung serotyping, or a serotype-specific UAD. CAP, community acquired pneumonia; cmPCR, conventional multiplex PCR; NP, nasopharyngeal; rmPCR, real-time multiplex PCR; UAD, urine antigen detection.

Of the 4769 cases of all-cause CAP with at least one test for $S$. pneumoniae $\left(\mathrm{UAD}_{\mathrm{SPN}}, \mathrm{UAD}_{\mathrm{PCV} 13}\right.$, sputum culture, or blood culture) (figure 1B), 1616 had NP swabs for viral studies. Of these, $14.8 \%(240 / 1616)$ were identified as $\mathrm{CAP}_{\mathrm{spn}}$ (figure 1B). Of the $\mathrm{CAP}_{\mathrm{spn}}$ cases, 161 (67.1\%) were non-bacteremic $\mathrm{CAP}_{\mathrm{Spn}}$, and $79(32.9 \%)$ were bacteremic $\mathrm{CAP}_{\text {Spn }}$ (figure 1A). The sensitivity for $S$. pneumoniae detection of $\mathrm{CAP}_{\mathrm{Spn}}$ using lytA and cpsA real-time PCR on NP swabs was significantly $(\mathrm{p}<0.0001)$ less than the modified gold standard at $34.17 \%(82 / 240)$ (table 1). It should be noted that $29 \mathrm{NP}$ swabs were positive in CAP cases not identified as pneumococcal disease (figure 1B), resulting in a lower post-test probability than observed in CAP cases where all pneumococcal tests were performed.

Of the NP swabs identified as $S$. pneumoniae positive by lytA/cpsA PCR from $\mathrm{CAP}_{\mathrm{Spn}}$ cases where all four pneumococcal tests were performed, a serotypeable result was obtained in $88.2 \%$ (30/34). The four non-typeable results originated from samples where lytA and cpsA values were near the detection limit (all Ct values were above 35), suggesting a bacterial load falling below the serotypeable range. ${ }^{9}$ For the serotypeable results, $100 \%$ concordance was observed compared with $\mathrm{UAD}_{\mathrm{PCV} 13}$ or Quellung serotyping of $S$. pneumoniae isolates. The identified serotypes included: $3(\mathrm{n}=10) ; 7 \mathrm{~F} / \mathrm{A}(\mathrm{n}=6) ; 22 \mathrm{~F} / \mathrm{A}(\mathrm{n}=3) ; 19 \mathrm{~A}(\mathrm{n}=2)$; $16 \mathrm{~F}(\mathrm{n}=2)$ and one each for serotypes $6 \mathrm{~A}, 6 \mathrm{C}, 9 \mathrm{~N} / \mathrm{L}$, $12 \mathrm{~F} / \mathrm{A} / \mathrm{B} / 44 / 46,13,23 \mathrm{~F}$ and $35 \mathrm{~B}$. The two non-CAP positive NP swabs were identified as serotypes 3 and 22F.

Of the positive NP swabs in CAP cases having received any one of the pneumococcal tests, a serotypeable result was obtained in $93.9 \%(77 / 82)$ (figure 2$)$. The four non-serotypeable results from NP positive in $\mathrm{CAP}_{\text {Sp }}$ cases as well the single non-typeable result from the $\mathrm{CAP}_{\mathrm{Spn}}$ case were all below the limit of detection for the PCR-based

Table 1 Summary of NP swab results compared with the modified gold standard among hospitalised adults with CAP

\begin{tabular}{lll}
\hline & NP swab PCR detection* versus & \\
\cline { 2 - 3 } Statistic & $\begin{array}{l}\text { CAP all tests十 } \\
\text { (n=434) }\end{array}$ & $\begin{array}{l}\text { CAP any tests } \\
\text { (n=1616) }\end{array}$ \\
\hline Sensitivity, \% $(95 \% \mathrm{Cl})$ & $35.42(25.92$ to 45.84$)$ & $34.17(28.17$ to 40.17$)$ \\
Specificity, \% $(95 \% \mathrm{Cl})$ & $99.41(97.88$ to 99.93$)$ & $97.89(97.13$ to 98.65$)$ \\
Positive likelihood ratio $(95 \% \mathrm{Cl})$ & $59.85(14.64$ to 244.67$)$ & $16.21(10.86$ to 24.20$)$ \\
\hline Negative likelihood ratio $(95 \% \mathrm{Cl})$ & $0.65(0.56$ to 0.75$)$ & $0.67(0.61$ to 0.74$)$ \\
\hline Pretest probability, \% $(95 \% \mathrm{Cl})$ & $22.12(18.30$ to 26.32$)$ & $14.85(13.12$ to 16.59$)$ \\
\hline Positive post-test probability, \% $(95 \% \mathrm{Cl})$ & $94.37(86.84$ to 101.90$)$ & $73.87(65.70$ to 82.05$)$ \\
\hline Negative post-test probability, \% $(95 \% \mathrm{Cl})$ & $15.58(12.02$ to 19.15$)$ & $10.50(8.95$ to 12.05$)$ \\
\hline
\end{tabular}

*Detection of Streptococcus pneumoniae in NP swabs was performed by real-time PCR targeting lytA and cpsA, and results were compared against modified gold standards in CAP cases where all ${ }^{\dagger}$ or any ${ }^{\ddagger}$ of the following tests were performed: sputum culture, blood culture, panpneumococcal urine antigen detection $\left(\mathrm{UAD}_{\mathrm{Spn}}\right)$ and/or a 13-valent pneumococcal conjugate vaccine serotype-specific $U A D\left(U A D_{\mathrm{PCV} 13}\right)$. $\mathrm{CAP}$, community-acquired pneumonia; NP, nasopharyngeal; UAD, urine antigen detection. 
serotyping methods. ${ }^{9}$ When detected, all serotypes were concordance with results obtained by Quellung or PCR-based serotyping on cultured $S$. pneumoniae isolates or those obtained from $\mathrm{UAD}_{\mathrm{PCV}_{13}}$ (figure 2). In addition, these serotypes and those obtained from NP-positive non- $\mathrm{CAP}_{\mathrm{Spn}}$ cases all showed predominance for serotypes $3,7 \mathrm{~F}, 19 \mathrm{~A}$ and $22 \mathrm{~F}$, as previously noted in this patient population. ${ }^{16}$

\section{DISCUSSION}

Laboratory tools for the detection and serotyping of $S$. pneumoniae are important to monitor the impact of pneumococcal vaccines, yet due to the high cost of disease surveillance, few studies go to the extent of comparing laboratory methods to clinical cases defined from CAP patient with pneumococcal disease. Compared with cases meeting the CAP definition, and with laboratory identification of $S$. pneumoniae from either culture-based or antigen-based laboratory tests, the performance of PCR-based detection and serotyping of $S$. pneumoniae from NP swabs collected for viral studies were evaluated. Overall, the sensitivity for $S$. pneumoniae detection was poor, but when positive, the subsequent PCR-based serotyping was concordant to results obtained with traditional serotyping or a serotype-specific UAD.

The poor sensitivity of PCR-based detection from NP swabs collected for viral studies noted in this study may simply be a reflection of its comparator. The performance of NP swab PCR was compared against $\mathrm{CAP}_{\mathrm{Spn}}$ cases receiving four other pneumococcal detection tests, each of which with its own advantages and limitations. For example, it is known UAD can remain positive for weeks after resolution of CAP, and UAD is less likely affected by factors such as antibiotic exposure compared with sputum or blood cultures. ${ }^{20-25}$ Poor sensitivity was also seen with quantitative lyt $A$ PCR on NP aspirates from patients with $\mathrm{CAP}$, and an association was observed between $S$. pneumoniae DNA concentrations and disease severity, onset of symptoms and host factors. ${ }^{26}{ }^{27}$ In this study, the sensitivity for S. pneumoniae detection using PCR from NP swabs was poor regardless of disease severity (ie, sensitivity for bacteremic $\mathrm{CAP}_{\mathrm{Spn}}$ was $32.4 \%(22 / 68)$ and $42.9 \%$ $(12 / 28)$ for bacteremic $\mathrm{CAP}_{\text {Spn }}$ when compared with CAP cases tested with all pneumococcal tests). It should be noted, however, that approximately $80 \%$ of patients with $\mathrm{CAP}_{\mathrm{Spn}}$ received antibiotics within 8 hours of admission to reduce severe outcomes, and the average time between NP swab collection was 2 days, suggesting that the sensitivity of $S$. pneumoniae detection by PCR might have been unfairly compromised. ${ }^{24} \mathrm{NP}$ swab detection might also have been affected by the time between collection, onset of symptoms and the progression of disease, which prompted hospital admission. Since only one swab was taken per case, the effects of antibiotics or collection time could not be assessed. Future studies should consider the effects of possible confounding factors such as the time of collection, prior antibiotic, progression of disease and host factors such as comorbidities or immunosuppression. ${ }^{24-27}$

While this study evaluated the performance for $S$. pneumoniae detection by PCR in NP swab collected for viral studies, it was recognised that other specimen types, such as oral and oropharyngeal swabs, were previously shown to perform better than sampling from the nasopharynx to assess pneumococcal colonisation. ${ }^{28-30}$ Since the performance of NP swab PCR is affected by the results of its comparator, a future studies would benefit from a direct comparison between PCR-based detection of pneumococcal DNA in NP swabs and concurrent quantitative $S$. pneumoniae from culture swabs collected from the nasopharynx. ${ }^{31}$ Other studies have also suggested broth enrichment prior to lytA real-time PCR can enhance the detection of $S$. pneumoniae, particularly for low density colonisation. ${ }^{32}$ In this study, culture from the NP swab was not possible due to the formulation of the universal transport medium, which includes antimicrobials to minimise bacterial and fungal contamination. Other formulations are available that enable the recovery of both bacteria and viruses. ${ }^{28}$

To determine the true colonisation rates in the presence and absence of pneumococcal disease, compliance to testing using all laboratory methods would also need to be improved. Of the 4769 CAP cases, only a subset $(\mathrm{n}=3851)$ had a pneumococcal test performed, and of these, a smaller proportion had a paired NP swab. This limitation lead to smaller number of CAP cases used as a comparator to assess the NP swab PCR performance. As many tests are not performed routinely in clinical laboratories with the empiric antibiotic treatment of CAP, the best estimation of the contributions of $S$. pneumoniae to CAP during active surveillance is in individuals who had radiography confirmed $\mathrm{CAP}$ and the combined use of several laboratory tests (sputum culture, blood culture and urine for two different antigen detection tests). Of 621 cases where all four tests were performed, only 434 (70\%) had an NP swab performed. Following a positive PCR in CAP cases where all pneumococcal tests were performed, the post-test probability of pneumococcal disease was $94 \%$. The negative post-test probability remained high at approximately $16 \%$ due to the poor sensitivity of NP swab PCR. When NP swab PCR was compared with a less stringentmodified gold standard (ie, CAP cases receiving any of the pneumococcal test), the number of cases increased to 1616. Again, the sensitivity was poor, and the specificity and positive post-test probability decreased with 29 positive results obtained solely by NP swab PCR. The possibility of false positive NP PCR results was not likely since detection relies on two genetic targets (lytA and $\operatorname{cps} A$ ), and more likely represented colonisation in the absence of disease, or colonisation in patients with CAP where pneumococcal disease failed to be identified by the other detection methods. With the noted poor sensitivity of NP swab PCR, it would be expected that the detection of $S$. pneumoniae colonisation be compromised in both presence and absence of disease. In this study, S. pneumoniae 
colonisation in CAP cases without pneumococcal disease might be between $0.6 \%(2 / 338)$ and $2.1 \%(27 / 1376)$, depending on the comparator (figure 1). If these rates of colonisation in healthy individuals are truly underestimated, the post-test probabilities and other performance characteristics could also be affected. ${ }^{33} 34$ The rates of $S$. pneumoniae colonisation are not well defined in older adults compared with children, but recent studies using lytA and cpsA PCR recently showed values between 5\% and $11 \%$ for NP swab PCR, and up to $28 \%$ with other specimen types, suggesting that colonisation rates in the absence of disease may also be underestimated..$^{28} 30$

While the sensitivity of the NP swab PCR may require optimisation, the subsequent use of PCR-based serotyping provides valuable information for surveillance. As seen for culture and UAD results in figure 2, PCR-based serotype deduction from NP swabs showed predominance for serotypes 3, 7F, 19A and 22F. This serotype trend mirrored the distribution previously reported from the CIRN SOS Network, and justifies the use of a algorithm-based approach using lytA and $c p s A$ real-time PCR for $S$. pneumoniae detection, followed by cmPCR and rmPCR. ${ }^{916}$ Of note, all PCR-based serotyping results were concordant to Quellung-based serotyping on S. pneumoniae isolates or the previously validated serotype-specific UAD. ${ }^{5}$ As expected, cmPCR and rmPCR-based serotyping was not as sensitive as $S$. pneumoniae detection using lyt $A$ and cpsA real-time PCR, resulting in a small number of non-serotypeable results. ${ }^{9}$

Overall, PCR-based detection and serotyping of S. pneumoniae add to the repertoire of epidemiological tools for pneumococcal surveillance, without the need for culture and isolation of the organism. Despite the need to improve sensitivity, PCR-based detection and serotyping of S. pneumoniae from NP swabs collected for viral studies showed high specificity and serotypes mirrored trends obtained by traditional methods. Many laboratories archive NP swabs for respiratory virus surveillance like influenza virus, and as such, this specimen type would be readily be available to rapidly monitor serotype distribution following changes in pneumococcal vaccine programmes. PCR-based detection and serotype deduction of S. pneumoniae colonisation from NP swabs offers value for pneumococcal surveillance.

\section{Author affiliations}

${ }^{1}$ Canadian Centre for Vaccinology (CCfV), IWK Health Center, Nova Scotia Health Authority (NSHA), and Dalhousie University, Halifax, Nova Scotia, Canada ${ }^{2}$ National Microbiology Laboratory (NML), Winnipeg, Manitoba, Canada

Correction notice This paper has been amended since it was published Online First. Owing to a scripting error, some of the publisher names in the references were replaced with 'BMJ Publishing Group'. This only affected the full text version, not the PDF. We have since corrected theseerrors and the correct publishers have been inserted into the references.

Acknowledgements The authors would like to thank the investigators of the CIRN SOS Network, including: Melissa Andrew (Dalhousie University, Halifax, Nova Scotia), Duncan Webster (Saint John Regional Hospital, Saint John, New Brunswick), Sylvie Trottier and Guy Boivin (Centre Hospitalier de Québec-Centre Hospitalier de l'Université Laval, Québec, Quebec City), Louis Valiquette (Centre
Intégré Universitaire de Santé et de Services Sociaux de l'Estrie-Centre Hospitalier Universitaire de Sherbrooke, Sherbrooke, Québec), Makeda Semret (McGill University Health Centre, Montreal, Québec), Anne McCarthy (Ottawa Hospital General Campus, Ottawa, Ontario), Allison McGeer (Mount Sinai Hospital, Toronto, Ontario), Mark Loeb and Jennie Johnstone (McMaster University, Hamilton, Ontario), and William Bowie and Grant Stiver (Vancouver General Hospital, Vancouver, British Columbia). The authors would like to thank all CIRN SOS site monitors, as well as the staff at the Canadian Center for Vaccinology (CCfV), who were instrumental in the coordination, processing and data analysis of CAP and IPD specimens. In particular, the authors would like to acknowledge Ardith Ambrose, Donna Mackinnon-Cameron, Lingyun Ye, Li Li and Michelle Warhuus.

Contributors The CIRN SOS Network principal investigator (SAM) and site investigators were involved in the conception and design of the CIRN SOS Network that performed active for surveillance of CAP and IPD; SAM, JJL, TFH and ME supervised the CIRN SOS Network central laboratory; JJL, ALSL and HDG were involved in method development and molecular testing for the study. IM provided reference serological testing at the NML on S. pneumoniae isolates. All authors drafted the manuscript, interpreted the data and revised the manuscript critically for important intellectual content. All authors approved the final manuscript.

Funding This work was supported by the Public Health Agency of Canada (PHAC), the Canadian Institutes of Health Research (CIHR) (grant number 23338) and by an investigator-initiated research grant to SAMN and the Canadian Immunization Research Network (CIRN) from Pfizer Canada (grant number PF1001). No study sponsors were involved in the study design, specimen collection, analysis and interpretation of data, scientific writing or decision to submit the paper for publication.

\section{Patient consent Obtained.}

Ethics approval REB committees for each hospital of the CIRN SOS Network in five Canadian provinces.

Provenance and peer review Not commissioned; externally peer reviewed.

Data sharing statement This study represents a subset of the data collected by the CIRN SOS Network and can be accessed by academic researchers with permission from the principal investigator, SAM (shelly.mcneil@cdha/nshealth.ca).

Open Access This is an Open Access article distributed in accordance with the Creative Commons Attribution Non Commercial (CC BY-NC 4.0) license, which permits others to distribute, remix, adapt, build upon this work non-commercially, and license their derivative works on different terms, provided the original work is properly cited and the use is non-commercial. See: http://creativecommons.org/ licenses/by-nc/4.0/

(c) Article author(s) (or their employer(s) unless otherwise stated in the text of the article) 2017. All rights reserved. No commercial use is permitted unless otherwise expressly granted.

\section{REFERENCES}

1. Bogaert D, De Groot R, Hermans PW. Streptococcus pneumoniae colonisation: the key to pneumococcal disease. Lancet Infect Dis 2004;4:144-54.

2. O'Brien KL, Wolfson LJ, Watt JP, et al. Burden of disease caused by Streptococcus pneumoniae in children younger than 5 years: global estimates. Lancet 2009;374:893-902.

3. McNeil SA, Qizilbash N, Ye J, et al. A retrospective study of the clinical burden of hospitalized all-cause and pneumococcal pneumonia in Canada. Can Respir J 2015.

4. Bettinger JA, Scheifele DW, Kellner JD, et al. The effect of routine vaccination on invasive pneumococcal infections in Canadian children, immunization monitoring program, active 2000-2007. Vaccine 2010;28:2130-6.

5. Bonten MJ, Huijts SM, Bolkenbaas M, et al. Polysaccharide conjugate vaccine against pneumococcal pneumonia in adults. $N$ Engl J Med 2015;372:1114-25.

6. Demczuk WH, Martin I, Griffith A, et al. Serotype distribution of invasive Streptococcus pneumoniae in Canada after the introduction of the 13-valent pneumococcal conjugate vaccine, 2010-2012. Can J Microbiol 2013;59:778-88.

7. Kellner JD, Vanderkooi OG, MacDonald J, et al. Changing epidemiology of invasive pneumococcal disease in Canada, 19982007: update from the Calgary-area Streptococcus pneumoniae research (CASPER) study. Clin Infect Dis 2009;49:205-12. 
8. Lovgren M, Spika JS, Talbot JA. Invasive Streptococcus pneumoniae infections: serotype distribution and antimicrobial resistance in Canada, 1992-1995. CMAJ 1998;158:327-31.

9. Lang AL, McNeil SA, Hatchette TF, et al. Detection and prediction of Streptococcus pneumoniae serotypes directly from nasopharyngeal swabs using PCR. J Med Microbiol 2015;64:836-44.

10. Lang ALS, Gillis HD, Elsherif M, et al. Refining PCR-based serotyping for detection of vaccine-preventable Streptococcus pneumoniae. $J$ Epidemiol Res 2016;3:28-36.

11. Messaoudi M, Milenkov M, Albrich WC, et al. The relevance of a novel quantitative assay to detect up to 40 major streptococcus pneumoniae serotypes directly in clinical nasopharyngeal and blood specimens. PLoS One 2016;11:e0151428.

12. Njanpop Lafourcade BM, Sanou O, van der Linden M, et al. Serotyping pneumococcal meningitis cases in the African meningitis belt by use of multiplex PCR with cerebrospinal fluid. J Clin Microbio 2010;48:612-4.

13. Pernica JM, Moldovan I, Chan F, et al. Real-time polymerase chain reaction for microbiological diagnosis of parapneumonic effusions in Canadian children. Can J Infect Dis Med Microbiol 2014;25:151-4

14. Resti M, Moriondo M, Cortimiglia M, et al. Community-acquired bacteremic pneumococcal pneumonia in children: diagnosis and serotyping by real-time polymerase chain reaction using blood samples. Clin Infect Dis 2010;51:1042-9.

15. Strålin K, Herrmann B, Abdeldaim G, et al. Comparison of sputum and nasopharyngeal aspirate samples and of the PCR gene targets lytA and Spn9802 for quantitative PCR for rapid detection of pneumococcal pneumonia. J Clin Microbiol 2014;52:83-9.

16. LeBlanc JJ, ElSherif M, Ye L. Burden of vaccine-preventable pneumococcal disease in hospitalized adults: a Canadian Immunization Research Network (CIRN) Serious Outcomes Surveillance (SOS) Network Study. Open Forum Infect Dis 2016;3(suppl_1):909.

17. Facklam RR, Washington JA, et al. Streptococcus and related catalase-negative gram-positive cocci. In: Balows A, Hausler WJ, Hermann L, eds. Manual of Clinical Microbiology. 5th Ed Washington, DC: American Society for Microbiology, 1991:238-57.

18. Spellerberg B, Streptococcus BC, et al. In: Murray PR, Baron EL, Jorgensen JH, eds. Manual of clinical microbiology. $9^{\text {th }}$ Edition. Washington: USA American Society of Microbiology, 2007:412-29.

19. Austrian $R$. The quellung reaction, a neglected microbiologic technique. Mt Sinai J Med 1976;43:699-709.

20. Bartlett JG. Diagnostic tests for agents of community-acquired pneumonia. Clin Infect Dis 2011;52 (Suppl 4):S296-S304.
21. Blaschke AJ. Interpreting assays for the detection of Streptococcus pneumoniae. Clin Infect Dis 2011;52 (Suppl 4):S331-S337.

22. Murdoch DR, Laing RT, Cook JM. The NOW S. pneumoniae urinary antigen test positivity rate 6 weeks after pneumonia onset and among patients with COPD. Clin Infect Dis 2003;37:153-4.

23. Saukkoriipi A, Palmu AA, Jokinen $\mathrm{J}$, et al. Effect of antimicrobial use on pneumococcal diagnostic tests in elderly patients with community-acquired pneumonia. Eur J Clin Microbiol Infect Dis 2015;34:697-704.

24. Song JY, Eun BW, Nahm MH. Diagnosis of pneumococcal pneumonia: current pitfalls and the way forward. Infect Chemother 2013;45:351-66.

25. Said MA, Johnson HL, Nonyane BA, et al. Estimating the burden of pneumococcal pneumonia among adults: a systematic review and meta-analysis of diagnostic techniques. PLoS One 2013;8:e60273.

26. Alpkvist $\mathrm{H}$, Athlin $\mathrm{S}$, Nauclér $\mathrm{P}$, et al. Clinical and microbiological factors associated with high nasopharyngeal pneumococcal density in patients with pneumococcal pneumonia. PLoS One 2015;10:e0140112

27. Albrich WC, Madhi SA, Adrian PV, et al. Genomic load from sputum samples and nasopharyngeal swabs for diagnosis of pneumococcal pneumonia in HIV-infected adults. J Clin Microbiol 2014;52:4224-9.

28. Krone CL, Wyllie AL, van Beek J, et al. Carriage of Streptococcus pneumoniae in aged adults with influenza-like-illness. PLoS One 2015;10:e0119875.

29. Trzciński K, Bogaert D, Wyllie A, et al. Superiority of trans-oral over trans-nasal sampling in detecting Streptococcus pneumoniae colonization in adults. PLoS One 2013;8:e60520.

30. Esposito S, Mari D, Bergamaschini L, et al. Pneumococcal colonization in older adults. Immun Ageing 2016;13:2.

31. Satzke C, Turner P, Virolainen-Julkunen A, et al. Standard method for detecting upper respiratory carriage of Streptococcus pneumoniae: updated recommendations from the World Health Organization Pneumococcal Carriage Working Group. Vaccine 2013;32:165-79.

32. da Gloria Carvalho M, Pimenta FC, Jackson D, et al. Revisiting pneumococcal carriage by use of broth enrichment and PCR techniques for enhanced detection of carriage and serotypes. J Clin Microbiol 2010;48:1611-8.

33. Gunnarsson RK, Lanke J. The predictive value of microbiologic diagnostic tests if asymptomatic carriers are present. Stat Med 2002;21:1773-85.

34. Orda U, Gunnarsson R, Orda S, et al. Etiologic predictive value of a rapid immunoassay for the detection of group A Streptococcus antigen from throat swabs in patients presenting with a sore throat. Int J Infect Dis 2016;45:32-5. 